\title{
A Totally Self-Checking Dynamic Asynchronous Datapath
}

\author{
Jing-ling Yang \\ Department of Electrical and Electronic \\ Engineering. \\ The University of Hong Kong \\ ibyans@eee.hku.hk
}

\begin{abstract}
This paper investigates the inherent totally self checling (TSC) property of one ope of dynamic asynchronous datapath based on Differential Cascode Vollage Logic. (DCVSL). As a result, a totally self checkting dymamic asynchronous datapath architecture is proposed. It is siapler than other similar approsches and represents a new approach to fault tolerant design.
\end{abstract}

Keywords: Totally selfchecking, asmchronous datapath, differential cascode voltage switch logic, divider

\section{Introduction}

A totally self-checking, TSC, circuit is a circuit that has self-test and fault-secure property for a normal input set $\mathrm{N}$ and faulty set F [1]. A TSC circuit should consist of a TSC functional part and a TSC checker. The inputs and outputs of the functional part are encoded using some suituble codes. The checker checks for code words st the outputs. If a non-code word is found, an error is indicated. In addition, a favit produced by a checker itself should also be detected. With the above characteristic, a TSC circuit well meets the requirements of online testing, where a fault, which is either permanent or transient, can be readily detected if the fault causes circuit malfunction.

It is known that delay insensitive circuits, one type of asynchronous control circuits, have the inherent selfcheck properties, that is all circuit activities cease (dead lock) with respect to single and multiple stuck-at faults at gate outputs $[2,3,4]$. Some authors have also showed that single stuck-at, stuck-closed, and stuck-open faults in most transistors of any Differential Cascode Voltage Logic (DCVSL) circuits result in either correct values or in loss of complementarity at outputs [S].

Typically a concurrent error detection asynchronous datapath with DCVSL computation block uses a TSC dual-rail code error indicator to check error from DCVSL computation blocks, and a non TSC RC time-out counter at each computation stage to indicate any asynchronous control deadlock error [6]. However this scheme is inefijicient because the use of non TSC RC time-out counter in every pipeline stages will lead to (1) large silicon. (2) TSC goal can not be achieved.

\author{
Chitr-sing Choy, Cheong-fat Chan, and \\ Kong-pong Pun \\ Department of Electronic Engineering \\ The Chinese University of Hong Kong \\ cschoy, cfchan and kppun@ee.cuhkedu.hk
}

This paper, on other hand, proposes a highly efficient TSC asynchronous datapath scheme with only one TSC duafrail code error indicator at its final stage. In other words, according to the new scheme, a TSC asynchronons dataputh with DCVSL computation block can be achieved simply by adding a dual-rail code error indicator at its final computation block.

\section{TSC Dynamic Asynchronous Datapath}

In a TSC synchronous circuit, input and outpot are coded as code words. If there is effective fault exists in the circuit, the circuit will output a non-code word that is distinguishable from the fault free circuit that outputs a code word.

Delay insensitive circuits have inherent TSC ability because they will deadlock with respect to single and multiple stuck-at faults at gate outputs. That is the presence of stuck-at faults may cause the circuits leave their normal cycle of states into a deadlock state. Here the conventional code words and non-code words are replaced by the circuits' valid states and deadlock tates. Further studies on properties and relations among these valid states and deadlock states, we found that the special deadlock property of a Latch-Free Dynamic Asynchronous Datapath (LFDAD) is well suited to implement TSC design.

\subsection{Latch-Free Dynamic Asynchronous Datapath}

DCVSL circuits have two interesting behavion. (1) During the precharge phase, input data has no effect on the output value. That means, Do matter what value of data appears at the input lines, the outputs (F and $\bar{F}$ ) will be precharged to low. (2) During the evaluation phase, DCVSL gate begins evaluation as soon as the input data are valid $(01$ or 10$)$. When the input data are not valid (00), the DCVSL gate remains at the precharge state.

These two behaviors lead to two advantage of a dynamic asynchronous pipeline over it static counterpart, nameły latch free and simpler handshake protocol and readiness to implement a TSC scheme. 
A LFDAD can work in the following fashion. First, after initialization, a current stage is allowed to go into the evaluation phase and remains in the evaluation phase (Hold) until the next stage has finished evaluation. Second, the current stage goes into the precharge phase when the next stage finishes evaluation, and ends the precharge phase when the second next stage finishes evaluation.

The handshake cell is a domino-style logic cell, see Figure 1. Figure 2 shows its timing diagram.

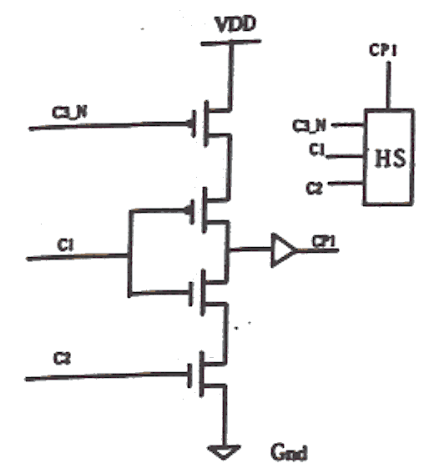

Figure 1: The Handshake Cell

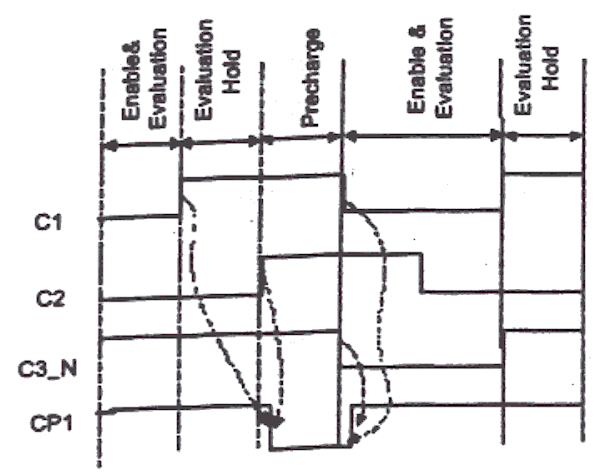

Figure 2: Operation Phase of the Handshake Cell

In Figure 1 and Figure 2, C1, C2 and $\mathrm{C3}$ are completion signals from stagel, stage 2 and stage 3 of a pipeline respectively, $\mathrm{C} 3$ _N is the compliment of $\mathrm{C} 3$.

The operation of pipeline stage is divided into 2 phase that include 3 steps: Evaluation phase, which includes Enable \& Evaluation and Evahation Hold two internal steps, and Precharge phase. In the Enable \& Evaluation step, the current stage processes the valid data at the input. After the current stage has finished the evaluation, it will enter the Evaluation Hold step. In this step, the input data may become invalid but the output should be held for the whole Enable \& Evaluation step for the next stage. After that, the current stage will enter the precharge phase that is necessary for DCVSL. Up to now the current pipeline stage has completed one complete cycle and goes into Enable and Evaluation step of next cycle waiting for a new valid data to eppear at the input.

Comparing with the handshake arrangement of Williams [7], this handshake cell is fuster toe to its protocol that allows he precharge signal be removed before the arrival of a valid data. Comparing with the handshake arrangement of Matsubarn's [8], although both protocols allow the precharge signal be removed before the arrival of a valid date, the handabake cell is faster due to its simplicity. And more importantly, this handshake protocol is robust becanse it hes no timing assumption that make it readiness to TSC implement, while Matsubara's pipeline has to satisfy the precharge width requirement.

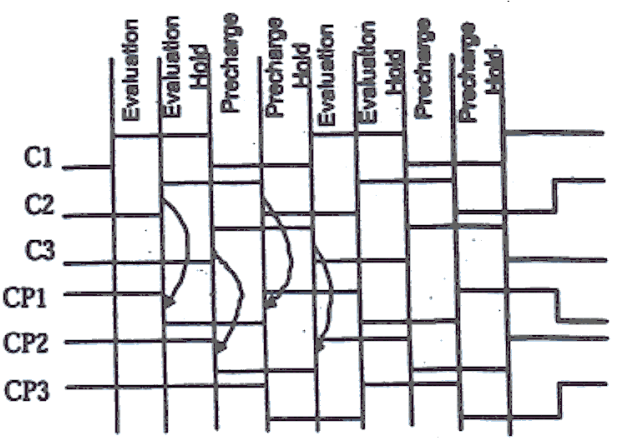

(a) Willians' Pipeline Handshalve Tuming

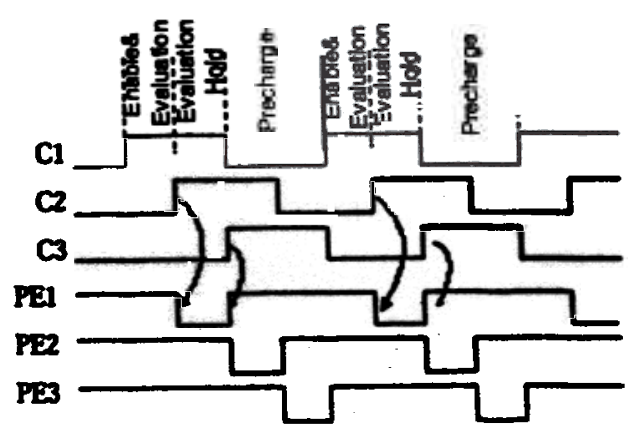

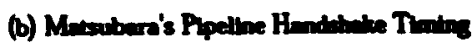

Figure 3: Signal Graphs of Different Handshake Cells

Figure 4 shows the implementation of a pipeline using the handshake cell. Each pipeline stage is composed of a dynamic DCVSL function block, completion detector and handshake cell. The completion detector indicates validity or absence of data at the outputs of the associated function. Each pair of data is checked by an XOR gate, and then uses a Celement to generate the final completion signal. 


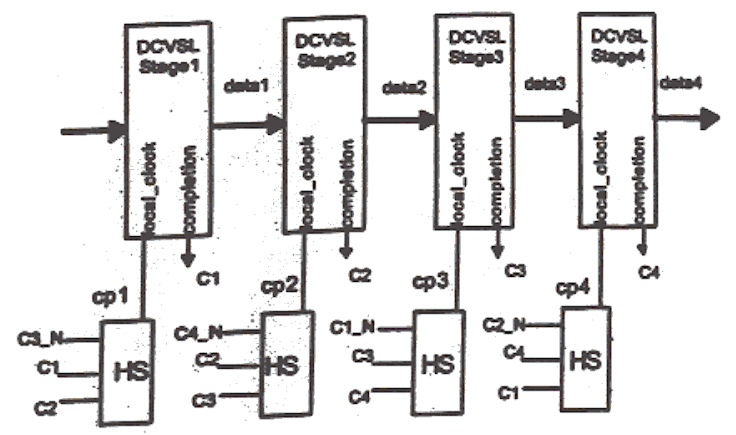

Figure 4: Structure of the Pipeline

\subsection{Inberent TSC Abilities on LFDAD}

To achieve concurrent error detection in a typical asynchronous datapath with DCVSL computation block, the most conventional method is treating them as two parts, using a TSC dualrail code error indicator to check error from DCVSL computation blocks, and a non TSC RC time-out counter at handshake circuits of each computation stise to indicate the asynchronous control deadlock error [6]. But the use of non TSC RC time-ont counter will lead to both large silicon and TSC goal can not be achieved.

Each pipeline stage of the LFDAD showed in Figure 4 has the following special properties. (1) Work in cycles consisting of three sequential steps, they are Enable \& Evaluntion, Evaluation Hold and Precharge. Enable \& Evaluation carries out computation according to input, Evaluation Hold keeps the output when next stage carries out computation, and then it goes into the precharge step. (2) A Fpeline stage is one step lagging behind its former stage or one step in advance of its later stage. (3) For each pipeline stage an effective error in its completion signal has no influence on its later stages' control signal, see Figure 2.

The combined effect of these behaviors is: if a stage has an effective error, its following stages will continue to work until they reach the same phase as the error stage. This is the very property to be used to simplify the TSC scheme of LFDAD.

Explore in more detail, we found that: (1) If the error stage is in the Precharge phase, because a DCVSL circuit in the precharge phase does not respond to any input data, jts following stages will continue to work until they also finish the precharge phase. (2) When the error stage is in the Evaluation phase, its following stages will continue going to the evaluation phase. Because in the evaluation phase, DCVSL gate begin evaluation as soon as the input data are valid, if its former stage cannot finish evaluation, the following stages remaining in the precharge phases.
Thus we can conclude that for those effective errors either in the evaluation phase or in the precharge phase, their ultimately effects are leaving its following stage in the precharge phase. This conclusion applies to stuck-at, stuck-closed, and stuck-open favits in most transistors of any DCVSL circuits and stuck-at fault handshake control signal.

So, to implement a TSC LFDAD, the only thing we need to do is adding a TSC dualrail code checker at its final stage.

\subsection{A TSC Scheme for LFDAD}

Figure 5 shows a proposed top-level structure of a TSC LFDAD. The TSC dual-rail checker can be a multiple input dynamic dual-rail XOR gate. The circuit works in this way: if the acquired output is correct, the data-checker outputs a dual-rail signal, otherwise if the acquired calculation output is not correct, data-checker outputs either a 00 or 11 signals. Or, if the pipeline has been stopped, the error-indicator pair will signal 00 .

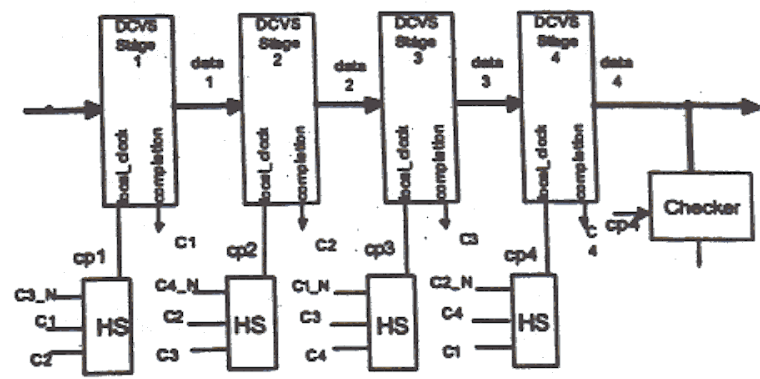

Figure 5: Top Level Structure of the TSC FLDAD

\subsection{TSC Analysis of other Similar Approaches}

For dynamic asynchronous datapath design, two approaches are popular. One is traditional latch based design [6], and the other is latch free design $[7,8,9]$.

An error detection scheme for a latch based design is demonstrated in [6]. In this scheme the whole datapath is treated as two parts, computation block and control block. A TSC two-rail code error indicator is used to check error from DCVSL computation blocks, and a non TSC RC time-out counter at each computation stage's handshake circuits is used to signal the asynchronous control deadlock error. But the use of non TSC RC timeout counter will lead to both large silicon and TSC goal cannot be achieved.

The TSC LFDAD design introduced in this paper belongs to latch free type design. In this approach, because of the exceptional indication of its deadlock state, the TSC goal can be efficiently realized by adding a TSC dual-rail code checker at its last stage. 
For other schemes of latch free approach, no TSC schemes have bein proposed. But for those schemes that have made timing assumption in their handshake protocol design, such as [9], the TSC goal may be hard to realize.

\section{A Bedign Case Study}

This section presents a highly efficient 8bit TSC asynchronous divider design. This design aims at verifying the feasibility and the hardware efficiency of the proposed TSE scheme.

Using the TSC scheme proposed, an \&-bit TSC asynchronous divider has been designed. Figure 6 shows the top-level structure of the TSC asynchronous divider with a dual-rail code checker.

To check dual-rail code, a dynamic dual-rail code checker (DDCC) is added at the output of the last stage. Its circuit is showed in Figure 7 . The output $(Z, \bar{z})$ are complementary if all of the input pairs are complementary, and if any input pair is 0,0 or 1,1 the outputs $(Z, \bar{z})$ take on that same value. If the circuits have stop working, the outputs $(Z, \bar{z})$ will be kept in 0,0 . Note that the DDCC itself is TSC.

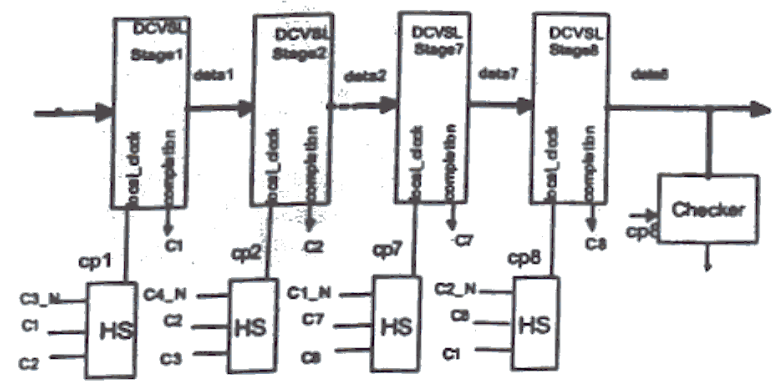

Figure 6: Top Level Structure of the TSC Divider

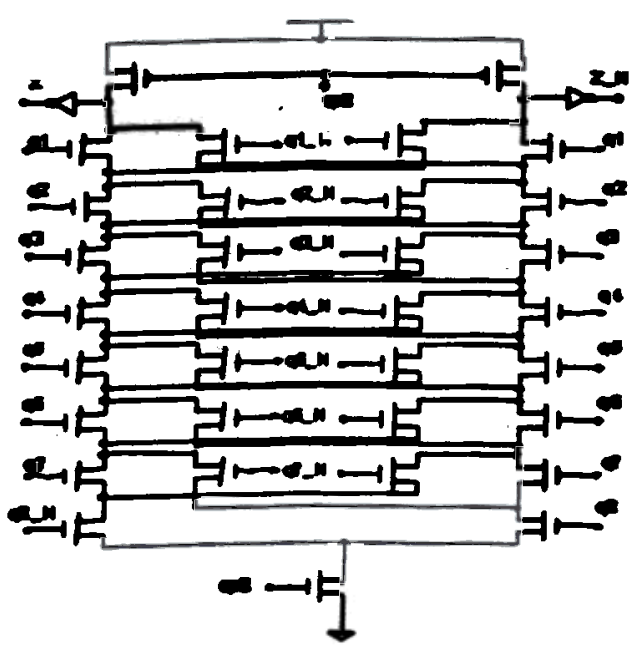

Figure 7: Dynamic Dual-rail Data Checker
The DDCC showed in Figure 7 is actually an 8bit dynamic duatrail XOR gate. In layout implementation, we can compose it by using seven 2-input dynamic duat nil XOR gutes.

\section{Results}

We implemented an 8-bit TSC asynchronoms divider using AMS CMOS $0.6 \mathrm{~mm}$ technology. The chip size is about $1.66 \mathrm{~mm} \times 1.70 \mathrm{~mm}$. The design wes simulated with SPECTRA in typical conditions. Simulation result shows that the TSC asynchronous divider worts with a clock period of 8.5ns, (about $117 \mathrm{MHz}$ ), and the latency for \& bit quotient-digit generntion is about 19 is (about 52.6 MHZ). Figure 8 shows the simulation realt of: (a) Local clock signal from eight thgee (b) Completion signal from eight stages. (c) Computation results of the divider. (d) Reesilts from DDCC.

The manufactured chip was texted with MSS XI-60 Logic Master. The test results show that if the acguired quotient is correct, the DDCC outputs a duafrail signal, otherwise if the acquired quotient is not correct, DDCC outputs either $a 00$ signal or 11 signal. $O$, if the TSC divider has stopped, the duatoutput pair will siomal 00 .

\section{Fault Simulation}

To perform fault simulation two tools are noeded, one is pattern generation tool, and another is fault simulation tool. Right now, prevailing commercial simulator are designed for static synchronous circuit under permanem fault model, while we want a fault simulation tool used for dynamic asynchronons circuit under transient error model. Because of this reason, this paper mainly relies on the amalyzis to validate the TSC ability of the dynamic asynchronous datapath, fault simulation is done manually only on some selected error. The selocted errors are chosen for their representativeness sccording to:

1. inserted error type: 0 or 1 ,

2. error location: control signal or dnta signal,

3. error instances: before or after completion sigmal generation.

The error is added to an error point through a XOR gate, showed in Figure 9.

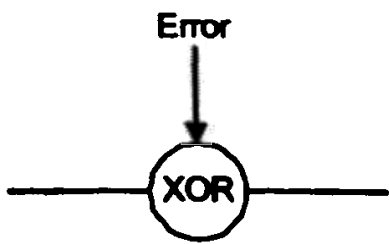

Figure 9: Error Insertion Point 
Error with lons duration time is inserted in a simulation cycle operated for 45ns. The selected transient errors give four kinds of results. They are: (1) Error has no effect on circuit. (2) Erroe be tolerated by circuit. (3) Error leads to delay of signal, bowever the circuits still work correctly because they tre delayinsersitive. (4) Error be detected.

With the above experiment, we confirm that the proposed TSC LFDAD scheme is secure against delay errors. It functions correctly under delayed signals caused by the transient errors, whereas in synchronous circuits, the delay faults will lead to circuit malfumction.

\section{Conclusion}

This paper analyses the inherent TSC abilitics of LFDAD, propose corresponding TSC sebeme. In addition, the proposed TSC structure is verified by a TSC 8-bit asynchronons divider design.

The fact that there is no undetected errors be found in the simulation and measurement indicate that the TSC ability of this type of design approach is sound.

\section{Acknowledgement}

The work reported is supported by a Hong Kong SAR government RGC, earmarked grant no. CUHK 4172/97E

\section{Reference}

[1] John Wakerly; " Error Detecting Codes, SelfChecking Circuits and Applications", Elsevier North-Hollend, lec., 1978.

[2] P. Beerel and T.Y. Meng, "Semi-Modularity and Testability of speed-independent Circuits", Integration, TheVLSIJ., Vol. 13, Sept 1992.

[3] VI. Varshavky, editor, " Self-timed Control of Concurrent Processes, Kluwer Academic Publishers, Dordrecht, The Netherlands, 1990.

[4] Michael J. Liebeit, and Neil Burgess, "Detecting Exitory Stuck-at Faults in Semimodular Asypchronons Circuits", IEEE Transaction on Computers, VOL.48. NO.4. April 1999.

[5] Nick Kanopoulos and Nagesh Vasanthavada, "Testing of Differential Cascode Voltage Switch (DCVS) Circuits", IEEE Journal of Solid-State Circuits, Vol. 25, No. 3, June 1990.

16] Divid A. Rennels and Hyeongil Kim, "Concurrent Error Detection in Self-timed VLSI", FTCS-24, Austin, Texas, June 15-17,1994.

[7] Jing-Ling Yang, Chiu-Sing Choy, Cheong-Fat Cheong, "A Self-Timed Divider Using a New Fast and Robust Pipeline Scheme", IEEE journal of Solid State Circuits. Vol. 36, No. 6, JUNE 2001.
[8] Ted E. Willams, Mark A. Horowiz, "A ZeroOverhend SelfTimed 160-ns 54b CMOS Divider", IBEE Joumal of Solid-State Circuits, VOL 26, No.11, pp. 1651-1661, November 1991.

[9] Gensoh Matsubara, Nobuhiro Ide, “A Low Power Zero-Overhead SelfTimed Division and Square Root Unit Combining a Single-Rail Stutic with a DoatRail Dynamic Circuits". Proceedimes of the Thind International Symposium on Advanced Research in Asynchronous Circuits and Syctem, 1997, pp. 198-209

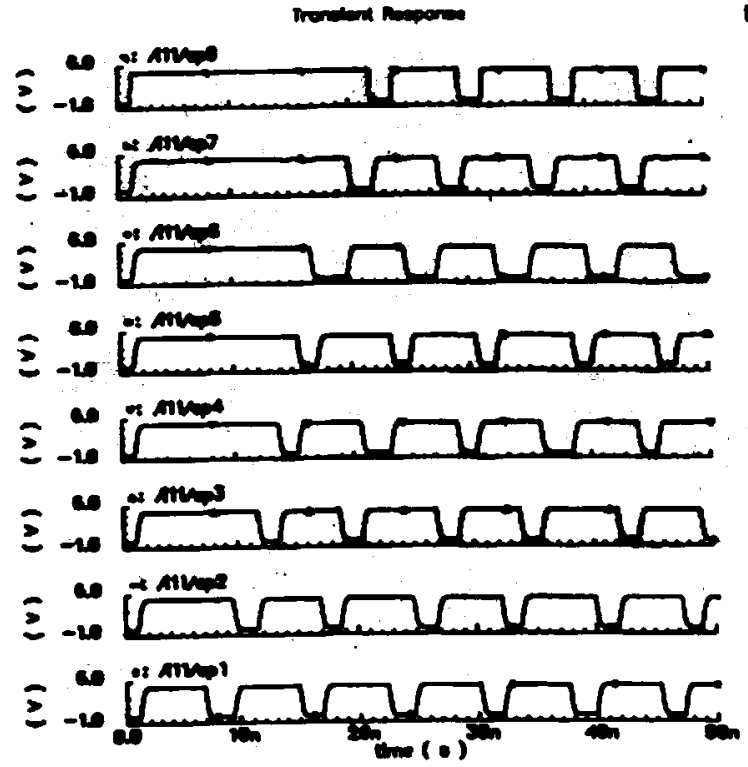

(a) Clock Sigal of each of the Eight Suges
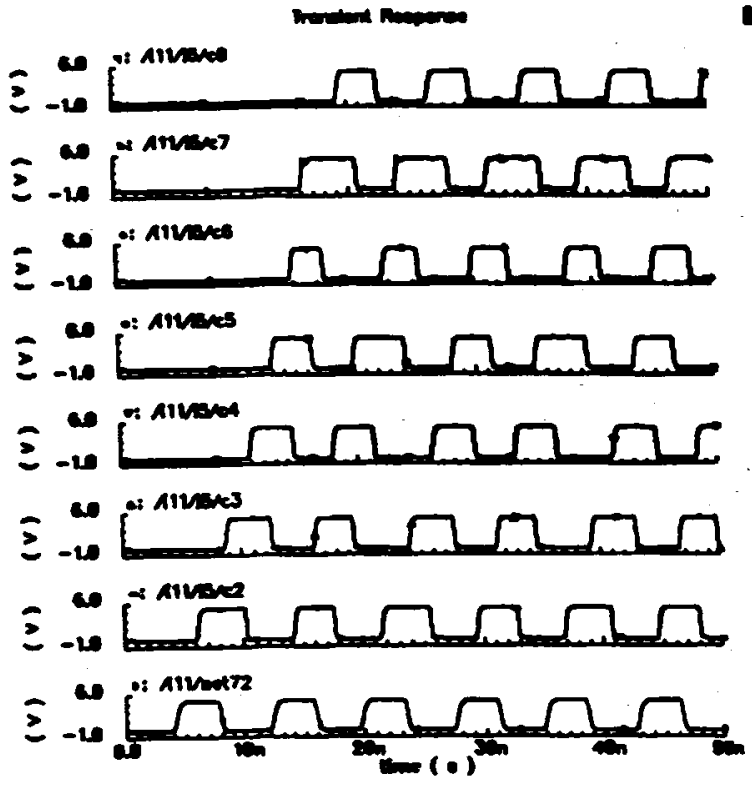

(b) Completion Signal from Eight Stages 

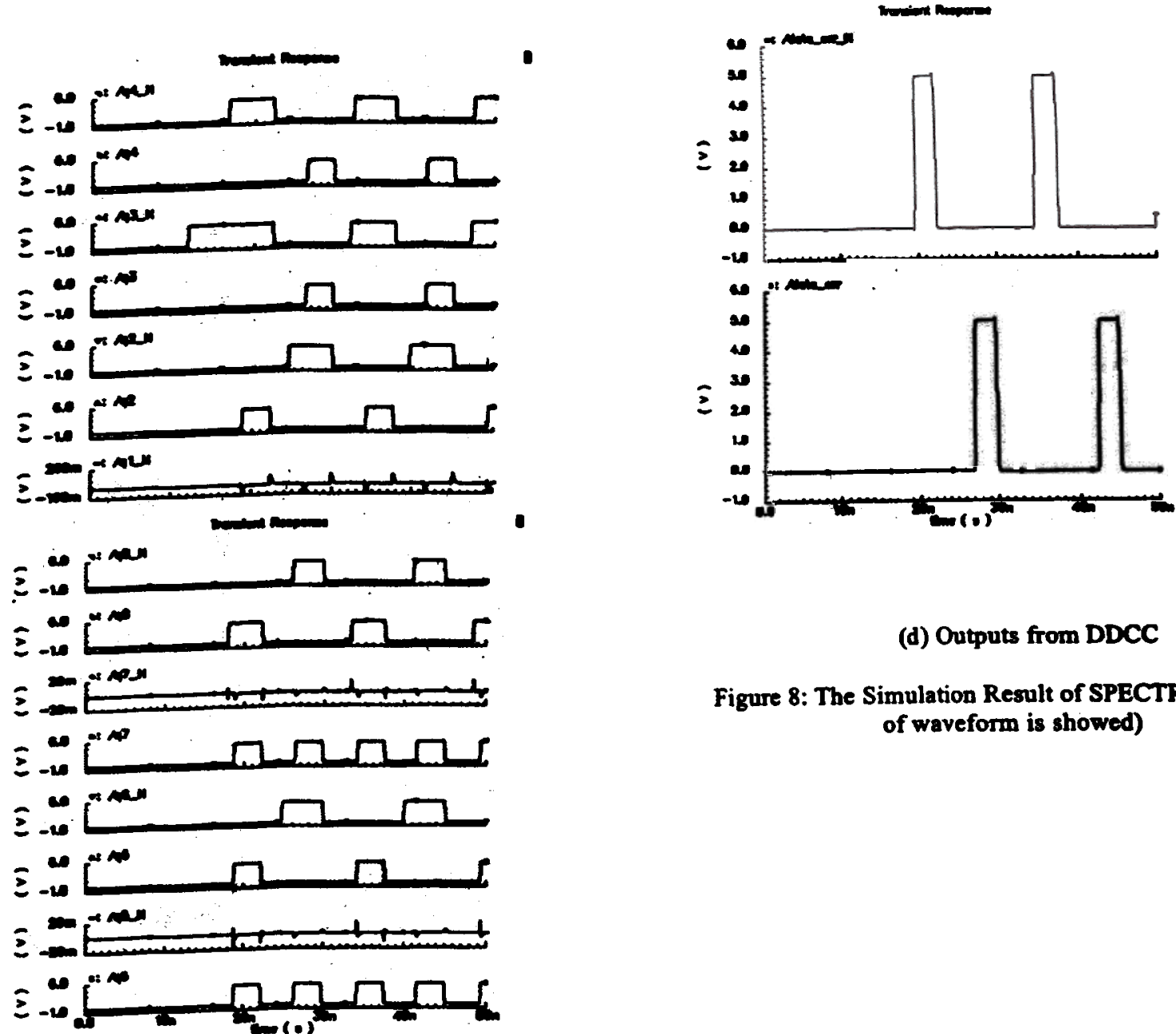

\section{(d) Outputs from DDCC}

Figure 8: The Simulation Result of SPECTRA (only part of waveform is showed)

(c) Computation Results of the Divider 